\title{
Gestão do Conhecimento e co-criação de valor em Serviços Informacionais
}

\author{
Murilo Mauro Silveira* \\ Gregório Varvakis*
}

Artículo recibido:

31 de marzo de 2020

Artículo aceptado:

27de junio de 2020

Artículo de revisión

\section{Resumo}

O objetivo do estudo é identificar como a GC (Gestão do Conhecimento) pode contribuir no processo de co-criação de valor em serviços informacionais. Justifica-se a pesquisa, ao ponto que, a GC oferece inúmeras vantagens em bibliotecas, com fins de mudar drasticamente suas necessidades e capacidades na prestação de serviços informacionais. A metodologia de matriz qualitativa e construtivista valeu-se do instrumento Knowledge Development Process - Constructivist (Pro-Know) para seleção da literatura, identificação/análise e formação de um Portfólio Bibliográfico (PB). A análise é baseada em Vargo

\footnotetext{
Programa de Pós Graduação em Ciência da Informação - Universidade Federal de Santa Catarina UFSC, Brasil willscut2@hotmail.com g.varvakis@ufsc.br 
e Lusch (2004), no tocante a GC e seus processos de troca de conhecimento (recursos operantes, dinâmicas, competências, aprendizagem e co-criação de valor. Sob a ótica dos serviços informacionais, resultou na discussão dos recursos operantes (informação e conhecimento) e sua gestão; o processo de troca (aprendizagem organizacional); as competências (dos profissionais); e o valor caracterizado neste nesse novo contexto (co-criação). Concluiu que a GC pode oferecer ferramentas para melhor prestar serviços informacionais, e não somente agregar valor, mais sim, cocriar valor, para que ambos (usuários e organização) se beneficiem neste contexto.

Palavras-chave: Gestão do Conhecimento; Serviços Informacionais

Gestión del conocimiento y co-creación de valor en los servicios de información

Murilo Mauro Silveira y Gregório Varvakis

\section{ReSUMEN}

El objetivo del estudio es identificar cómo la GC (gestión del conocimiento) puede contribuir al proceso de cocreación de valor en los servicios de información. La investigación está justificada, puesto que la GC ofrece numerosas ventajas en las bibliotecas con el propósito de cambiar drásticamente sus necesidades y capacidades en la provisión de servicios de información. La metodología de la matriz cualitativa y constructivista utilizó el proceso de desarrollo del conocimiento - instrumento constructivista (Pro-Know) para la selección de literatura, identificación / análisis y formación de un portafolio bibliográfico (PB). El análisis se basa en Vargo y Lusch (2004), con respecto a la GC y sus procesos de intercambio de conocimiento (recursos operativos, dinámicas, competencias, aprendizaje y cocreación de valor). Desde la perspectiva de los servicios de información, resultó en la discusión de recursos operativos (información y conocimiento) y su gestión, el proceso de intercambio (aprendizaje organizacional), las habilidades de los profesionales y el valor caracterizado en este nuevo contexto (cocreación). Se concluye que la GC puede ofrecer herramientas para mejorar los servicios 
de información, y no sólo agregar valor, sino más bien crearlo conjuntamente, de modo que tanto los usuarios como la organización se beneficien en este contexto.

Palabras clave: Gestión del conocimiento; Servicios de Información

Knowledge Management and co-creation of value in Information Services

Murilo Mauro Silveira and Gregório Varvakis

\section{Abstract}

The objective of this study is to identify how KM (Knowledge Management) can contribute to the process of co-creation of value in information services. The research is justified to the extent that KM offers numerous advantages in libraries in order to dramatically change their needs and capabilities in providing information services. The qualitative and constructivist matrix methodology resorts to Knowledge Development Process - Constructivist (Pro-Know), an instrument for literature selection, identification / analysis and formation of a Bibliographic Portfolio (PB). The analysis is based on Vargo and Lusch (2004), regarding KM and its knowledge exchange processes (operant resources, dynamics, competences, learning and co-creation of value). From the perspective of informational services, it resulted in the discussion of operant resources (information and knowledge) and their management; the exchange process (organizational learning), the skills (of the professionals), and the value characterized in this new context (co-creation). It is concluded that KM can offer tools to better provide informational services, not only adding value, but co-creating value, so that both (users and organization) benefit in this context.

Keywords: Knowledge Management; Informational Services 
$A_{\text {formação e comunicação, à transmutação dos conceitos de espaço e de }}^{\text {explosão informacional }}$ tempo convertidos na urgência do aqui e agora em dar resposta às necessidades dos clientes-utilizadores é uma realidade. O modelo atual de prestação de serviço de uma Unidade de Informação torna-se insustentável e obsoleto (Carvalho, Menezes e Domingues, 2012).

A literatura mostra que as diversas perspectivas sobre os serviços até hoje desenvolvidas desde os modelos de aferição da qualidade até a Lógica Dominante em Serviços (SDL), embora centrem as suas análises nas condições necessárias para haver participação do cliente utilizador na criação do valor do serviço revelam alguma insipiência no entendimento sobre o modo como este processo deve ser realizado. (Costa e Martins, 2012).

Assim, Vargo e Lusch (2004) propuseram alterar o atual padrão utilizado pelo Marketing totalmente voltado a venda de produtos ou bens de consumo para o de serviços. Sob a SDL, toda economia é uma economia de serviços e, dessa forma, deve estar orientada para os recursos operante (conhecimentos e habilidades) e para a co-criação de valor entre a empresa e o cliente.

Para Islam, Agarwal e Ikeda (2015) o conceito da criação de novos e inovadores serviços baseados na SDL leva em conta o conhecimento, as habilidades e os processos envolvidos na entrega do serviço. Atualmente, a lógica SDL surge como uma perspectiva onde o foco recai sobre os recursos operantes e intangíveis, baseados nas competências e no conhecimento dos atores em determinada organização, e é na interação que estes desenvolvem os processos de co-criação de valor.

Neste cenário, as Unidades de Informação devem redimensionar os serviços e as estruturas de apoio que permitam desenvolver e potenciar um maior grau de autonomia, competências e conhecimentos de modo a responder às necessidades de um novo cliente que surge como um protagonista ativo (Pérez Rodriguez e Milanés Guisado, 2008). A Gestão do Conhecimento (GC) é percebida como um modo potencialmente viável pelo qual as bibliotecas podem responder aos vários desafios que enfrentam em seu ambiente operacional em constante mudança (Sarrafzadeh et al., 2010: 199, citado por Balagué, Düren e Saarti, 2016). Isso porque, os usuários da biblioteca são cada vez mais exigentes com relação aos serviços.

Contudo, por meio de revisão de literatura, Ugwu e Ekere (2019) mostraram que os esforços de investigação anteriores nesta área voltados para abordagens GC há muito poucos estudos sobre práticas de GC em bibliotecas. Precedeu assim que os estudos sobre GC em serviço de biblioteca parece ser 
escasso, embora a GC ser considerada necessária neste contexto.

Neste cenário, questiona-se como a GC pode contribuir para que os serviços informacionais possam ser melhorados e adaptados as necessidades dos usuários por meio da co-criação de valor? Assim, o objetivo deste estudo é identificar como a GC pode contribuir no processo de co-criação de valor em serviços informacionais.

Para atingir os objetivos, é utilizada para discussão teórica a obra de Vargo e Lusch (2004), quanto aos aspectos relacionados à GC e seus processos de troca. Sob a ótica dos serviços informacionais, considerou-se recursos operantes (informação e conhecimento) e sua gestão; o processo de troca (aprendizagem organizacional); as competências (dos profissionais); e o valor caracterizado neste nesse novo contexto (co-criação).

$\mathrm{O}$ artigo pretende ser um contributo para a construção de um quadro conceitual dos processos de co-criação de valor dos serviços informacionais, considerando os conceitos desenvolvidos na literatura acadêmica sobre serviços informacionais e a gestão do conhecimento.

Justifica-se a pesquisa, ao ponto que, a GC oferece inúmeras vantagens em bibliotecas, com fins de mudar drasticamente suas necessidades e capacidades de prestação de serviços, entre seus usuários (Hadagali, 2012, citado por Islam et al., 2015).

No mundo dos negócios, a GC é aplicada principalmente para a vantagem competitiva, enquanto que no cenário biblioteca, GC é aplicada para melhorar a utilização dos recursos e para a prestação de serviços mais dinâmicos e eficazes (Ugwu e Ekere, 2019). Já Ugwu e Ekere (2018) afirmam que é necessário que estudos futuros se concentrar em investigar os efeitos da criação de valor e co-criação de valor em inovação para serviços de biblioteca.

O estudo delimita-se sobre a análise da literatura internacional sobre Gestão do Conhecimento e Serviços informacionais em artigos disponíveis no portal de periódicos da Coordenação de Aperfeiçoamento de Pessoal de Nível Superior (CAPES), entre os meses de junho a agosto de 2019.

\section{Gestão do Conhecimento e Gestão da Informação}

As organizações utilizam de informação e conhecimento para melhorar seu desempenho e obter vantagens competitivas. A gestão destes ativos é crucial para o melhor aproveitamento destes recursos. Valentim (2006), citado por Botelho, Monteiro e Valls (2007) enfatiza a importância de gerenciar eficientemente a informação e o conhecimento nos ambientes corporativos. Segundo o autor, a organização gera e compartilha a informação e o conhecimento 
e, por meio dessas ações, alimenta e retroalimenta uma estrutura organizacional, desde o planejamento até a execução propriamente dita, assim como, o processo decisório.

Considera-se necessário a Gestão da Informação (GI) nos processos que envolvem a Gestão do Conhecimento (GC). Antes de apresentar estes dois modelos de gestão, faz se necessário a conceituação destes no contexto organizacional. Para Valentim (2002), citado por Alavi, Kayworth, Leidner (2005), a GI é um conjunto de estratégias para identificar as necessidades informacionais, mapear os fluxos formais de informação nos diferentes ambientes da organização, assim como coleta, filtragem, análise, organização, armazenagem e disseminação da informação, objetivando apoiar o desenvolvimento das atividades cotidianas e a tomada de decisão em ambiente corporativo. Já a GC é o conjunto de estratégias para criar, adquirir, compartilhar e utilizar ativos de conhecimento, bem como estabelecer fluxos que garantam a informação necessária há tempo e formato adequados, a fim de auxiliar na geração de ideias, solução de problemas e tomada de decisão (Botelho, Monteiro e Valls, 2007).

No contexto organizacional é observado esta distinção, para Venkatesh e Kalpavalli (2014) no ambiente de GC, o papel da GI inclui não apenas o gerenciamento de tarefas tradicionais de gerenciamento de bibliotecas, mas também o uso eficaz da informação e do conhecimento armazenado em recursos primários, secundários e terciários. A GI inclui capacidades, bem como a adição de valor e oportunidades para atender desafios. A GC por sua vez, acrescenta experiência humana através do desenvolvimento de recursos humanos e sua gestão, é quase um passo à frente da gestão da informação.

Tais afirmações é corroborada por Botelho, Monteiro e Valls (2007) no sentido que existem similaridades entre GI e a GC, mas o último é mais amplo no seu âmbito e inclui (em particular), os aspectos de processo e de aprendizagem que não são as maiores preocupações de gestão de informação. Em comparação com o GI, a GC vai além do armazenamento e manipulação da informação. É uma tentativa de reconhecer o que é essencialmente um recurso enterrado na mente dos indivíduos, e revelá-lo como um ativo organizacional, que pode ser acessado e utilizado pela empresa (Johannsen, 2000).

A partir destas analise, pode-se afirmar que a GC e a GI representam duas disciplinas de gestão. Para Johannsen (2000), muitas são as semelhanças, mas também com uma série de diferenças significativas. $\mathrm{Na}$ verdade, as duas disciplinas são muitas vezes integradas na prática. Por exemplo, a construção de um banco de dados contendo experiências do projeto é essencialmente uma tarefa GI, mas também pode ser visto como um esforço da GC de transformar o conhecimento individual em coletivo. 
Até aqui, procurou-se identificar perspectivas, práticas, atitudes e respostas organizacionais para a gestão do conhecimento, incluindo como ela é conceituada, suas características principais, sua relação com a GI. Observou-se, portanto, sua contribuição para uma melhor gestão destas organizações que visam a disponibilização da informação por meio de seus serviços prestados. Contudo, mas especificamente, é relevante observar como a GC influencia a prestação destes serviços informacionais.

\section{Serviços informacionais e a Gestáo do Conbecimento}

A dinâmica de serviços de biblioteca está evoluindo com novas tendências, métricas para medir e avaliar o impacto de pesquisa, e os ecossistemas de análise orientada. Os serviços baseados na web estão mudando profundamente este cenário (Balaji et al., 2018). GC em bibliotecas é uma ferramenta para uma gestão sistemática. É baseada no conceito de que o controle do conhecimento precisa ser visto como um recurso importante na prestação de serviços de informação de alta qualidade nas bibliotecas (Balagué, Düren e Saarti, 2016).

Antes de adentrar nesta seara, cabe conceituar serviços informacionais. Para Wijnhoven e Kraaijenbrink (2008) trata-se da execução de serviços para ajudar as pessoas a entender o mundo, fornecendo ou tornando a informação relevante, útil e acessível. Um serviço de informação é capaz de: adquirir, agregar, exibir, processar, a criação e entrega de conteúdo de acordo com as necessidades do cliente, de modo a que os dados se tornam mais relevantes para ele.

Uma melhor representação sobre serviços informacionais pode ser observada em exemplos destes serviços. Para Miao (2001) o serviço de informação compreende pesquisa de literatura, monitoramento tecnológico, edição de boletim informativo, recorte de jornal, estudo de políticas e pesquisa de mercado, etc. Ainda segundo os autores, as coleções digitais em elevado crescimento, necessita de inteligentes ferramentas, como software de pesquisa, mecanismos de busca e mineração de dados. Estes são instrumentos facilitadores de um novo conceito de serviços.

Wijnhoven e Kraaijenbrink (2008) aprofundaram esta temática sobre a concepção de serviços informacionais. Para os autores, este tipo de serviço facilita a troca de bens de informação com ou sem transformar esses bens. Com o avanço das tecnologias, estes serviços ser tornaram disponíveis por equipamentos, contudo, os profissionais ainda têm papel na questão de gerenciá-los (design, operação, configuração).

Neste sentido, Venkatesh e Kalpavalli (2014) afirmam que todos os profissionais de biblioteca devem reformular e remodelar as ferramentas de 
gerenciamento de bibliotecas tradicionais e aplicar conceitos e práticas de GC para a prestação de melhores serviços em bibliotecas.

Ferguson, Hider, Lloyd (2008) observaram que os profissionais de serviços de informação têm vindo a assumir um maior papel na GC. Segundo os autores, em uma análise na literatura que aborda a oportunidade para profissionais de serviços informacionais, Rooi e Snyman (2006), citado por Ferguson, Hider e Lloyd (2008) sugere cinco grandes papéis para bibliotecários: facilitar um ambiente propício à partilha de conhecimentos; gerenciar a memória corporativa; atuar na transferência de mensagens instantâneas e habilidades relacionadas a um novo contexto ligados a processos de negócios e operações centrais; desenvolvimento de competências em informação corporativa; e, finalmente, a Gestão da Informação em um ambiente digital / eletrônico. Assim, observou-se o profissional da informação na tratativa da GC em serviços informacionais.

Fowell (2002) afirma que é cada vez mais complexa a natureza evolutiva do ambiente de informação das empresas e as perspectivas dos diferentes grupos de interessados apresentam uma série de desafios para os designers e gestores de recursos e serviços de informação. $\mathrm{O}$ autor estudou o impacto destes desafios para a concepção e gestão de recursos de informação ser úteis e utilizáveis.

Alguns estudos também demonstraram a necessidade da GC na melhoria dos serviços informacionais. Islam et al. (2015) retrataram que os resultados de sua pesquisa revelam que o uso e aplicação de GC em serviços de informação melhora o acesso aos recursos e serviços de informação, conhecimento e enriquecimento de profissionais da informação, aprimoramento do ambiente e cultura de conhecimento e o comportamento dos profissionais da informação.

Já White (2004), citado por Islam et al. (2015), argumentou que, no século 21, a GC foi, cada vez mais, se tornando uma ferramenta fundamental para ajudar a fornecer um serviço dinâmico e eficaz para usuários da biblioteca. Outros benefícios da aplicação da GC em bibliotecas incluem a promoção de relações dentro e entre as bibliotecas, entre bibliotecas e usuários, e o fortalecimento da rede de conhecimento que aumentou o ritmo dos fluxos de conhecimento (Shanhong, 2002). Ugwu e Ekere (2019) estudaram as atividades de GC em serviços de biblioteca com a finalidade de propor um modelo GC para a melhoria do serviço.

Assim, evidenciaram-se as vantagens de GC em serviços informacionais, mas questiona-se como esta gestão é operada nestas organizações? Os processos de GC em bibliotecas, e seus serviços são descritos por Ugwu e Ekere (2018), quando afirma que estes processos afetam a inovação de serviços de 
forma positiva. Seu estudo foi feito em biblioteca universitárias da Nigéria, e apontou que as três medidas no ciclo da GC, ou seja, captura de conhecimento / criação e compartilhamento de conhecimento / transferência e aplicação do conhecimento; foram encontrados e teve efeito positivo na escala em inovação de serviços em bibliotecas.

Para Ugwu e Ekere (2019), os processos de GC são: a identificação de conhecimento, aquisição de conhecimento, criação de conhecimento e disseminação do conhecimento. Segundo Ugwu e Ekere (2018), esses processos, inclui a aprendizagem sobre possibilidades de inovação, aprendizado sobre interface de usuário, aprendizado sobre as ferramentas tecnológicas para adotar e aprender sobre as necessidades dos usuários para compreendê-los.

Assim, percebe-se que o usuário é peça fundamental e o intento maior em pensar a melhoria dos serviços informacionais. A GC é uma forma de melhorar este contexto, que por meio do aprendizado, conforme Ugwu e Ekere (2019), pode colaborar na melhor relação entre a organização biblioteca e o usuário, em um processo mútuo de agregação de valor, ou co-criação.

\section{Co-criaçáo de valor em Serviços Informacionais}

As bibliotecas, por serem em sua maioria sem fins lucrativos, ou, seu papel esta vinculado a uma organização que prestam serviços sem retorno monetário, enfrentam a questão de demonstrar valor para seus stakeholders. Para Pantry e Griffiths (2000), é necessário que esta instituição perceba que seus serviços de informação não são simplesmente uma sobrecarga de custo, mas que, podem contribuir para a retaguarda organizacional. A contribuição pode ser facilmente observada em termos de valor agregado. O serviço deve pelo menos ser capaz de mostrar que está fornecendo mais valor. Um serviço de informação que demonstrar essa abordagem tem o direito de ser ouvido e respeitado por sua contribuição organizacional.

As bibliotecas precisam, cada vez mais, demonstrar valor por meio de serviços que agreguem valor aos usuários ou interessados. O profissional da informação é parte deste processo. De acordo com Dickinson (2004), este trabalhador é confrontado com o duplo desafio de demonstrar o valor do serviço de informações, e garantir serviços de informação de alta qualidade com recursos muito limitados. Para o autor, o desafio para o profissional de informação inclui: fornecer informação de alta qualidade e confiável, tornar os dados disponíveis através de interfaces relevantes, de fácil utilização; entregar novos serviços de valor agregado; ajudar a fornecer informações sobre o retorno a ser obtido através do investimento em tais serviços; e fazer desses serviços disponíveis serem de forma rentável a organização (Dickinson, 2004). 
Pensar a rentabilidade na prestação de serviços informacionais, é reconhecer o valor na prestação destes, e isso implica em perceber o quanto ele representa para o usuário. O usuário também é elemento chave neste processo, sua coparticipação na dinâmica da prestação de serviços, é essencial para que este possa ser melhorado, do ponto de vista que seus intentos possam ser descritos e absorvidos pelo prestador de serviços.

Para Van Deventer e Snyman (2004), tradicionalmente bibliotecas e clientes nem sempre percebem que eles são co-responsáveis pelos serviços e produtos, e que são apoiados financeiramente por ambas as partes interessadas. Segundo os autores o modelo original deve ser adaptado e colocar o cliente em pé de igualdade. Davis e Somerville (2006) descobriram em sua pesquisa, que os profissionais possuem sistemas pensados em construir contextos de apreciação e expansão necessários para co-criar valor em produtos e serviços inovadores em bibliotecas, e que a equipe de liderança liga isso simultaneamente com os processos de planejamento estratégico da organização.

Portanto, percebe-se que as bibliotecas devem integrar usuários e profissionais da informação de forma a co-criar valor em seus serviços informacionais. A GC colabora com este processo. Para Stoffle (1996) citado por Ahmad (2017), a GC deve ser visto como uma função primária de bibliotecas acadêmicas. Já Van Deventer e Snyman (2004) afirmam que medidas de sustentabilidade não são definitivamente sobre mostrar o valor financeiro para a organização ou somente demonstrando valor acrescentado para o cliente. Os gerentes de bibliotecas e seus serviços de informação precisam aceitar que a economia do conhecimento exige que todas as empresas, também bibliotecas e serviços de informação (mesmo centros de conhecimento) adotem novas maneiras de medir o seu crescimento para garantir a sustentabilidade a longo prazo.

Desta forma, a bibliotecas por meio da prestação de serviços de valor agregado, pode considerar a participação do cliente neste processo, e ir mais além, permitindo que este usuário também contribua de forma a agregar mais valor nos serviços, e assim, garantir sua sustentabilidade. Contudo, Ugwu e Ekere (2018) identificaram que é necessário que estudos futuros se concentrem em investigar os efeitos da criação de valor e co-criação de valor em serviços de biblioteca, de forma a visar a inovação destes serviços, utilizando as praticas de GC.

Percebe-se que a GC pode contribuir na melhoria e inovação de serviços informacionais em bibliotecas, de forma a permitir a co-criação de valor por parte dos interessados (organização e clientes). Não obstante, é necessário discutir este cenário, e as possibilidades de como a GC pode ser útil. 


\section{Metodologia De Pesquisa}

Quanto ao enquadramento metodológico, a abordagem do problema é qualitativa, pois, de acordo com Creswell (2014), a visão de mundo do pesquisador é que dá sentido às interpretações em que o mundo é representado, nesta pesquisa devotado à análise e interpretação das variáveis em relação a gestão do conhecimento e serviços informacionais.

A cosmovisão construtivista é a base filosófica em que os pesquisadores se orientam. De acordo com Roy (1993), essa perspectiva tenta contribuir com os participantes por meio de um conjunto de chaves, com base em objetivos e valores, identificando sistemas que ajudam a compreender contextos em que eles pretendem intervir.

Pesquisa-ação é o tipo de estratégia utilizada para investigação, considerando que os pesquisadores atuam e respondem como participantes para a evolução dos processos, isto é, os pesquisadores/participantes utilizam limitações e escolhas no processo de desenvolvimento da primeira fase do ProKnow-C.

O Knowledge Development Process - Constructivist (ProKnow-C) é o instrumento de intervenção utilizado nesta pesquisa. De acordo com Ensslin, Ensslin e Pinto (2013), objetiva construir conhecimento sobre um determinado tema a partir dos interesses e delimitações do pesquisador.

O ProKnow-C compõe-se de quatro fases de operacionalização: i) seleção de um Portfólio Bibliográfico (PB); ii) análise bibliométrica do PB; iii) análise sistêmica dos artigos do PB; e iv) definição da pergunta de pesquisa.

O processo de seleção do portfólio bibliográfico (primeira fase do ProKnow-C), utilizado nesta pesquisa, é realizado a partir de uma série de procedimentos pré-definidos, iniciados a partir do desejo do pesquisador em conhecer um rol de pesquisas científicas que representam em essência, determinado assunto. Assim, a partir de um processo sistematizado de filtragem e análise de aderência aos objetivos do pesquisador, torna-se possível selecionar um conjunto de artigos passam a compor o portfólio bibliográfico.

Para seleção do PB sobre o fragmento da literatura sobre Gestão do Conhecimento (GC) e serviços informacionais, seguiram-se os seguintes procedimentos: Primeiramente selecionaram-se as palavras chaves e montou-se o comando de busca ("knowledge management" and "information service*" or "library service"), para consulta em duas bases de dados (Web Of Science, Scopus). Depois de consultadas as bases, foram recuperados 111 artigos, dos quais seguiu-se os procedimentos de filtragem propostos pelo ProKnow-C. Assim foram excluídos: 24 artigos por estarem duplicados; 2 não disponíveis; 2 de acesso não aberto, e 56 não estavam adequados com a temática da pesquisa, a partir da leitura integral. Portanto, como resultado final, foi formado um portfólio composto de 27 artigos. 
Para análise dos dados, a discussão a respeito dos resultados utilizará como base a abordagem qualitativa de Creswell (2014). Cabe ressaltar que essa etapa consiste em uma avaliação subjetiva dentro desse processo. A partir de então, é realizada a avaliação dos dados para posterior discussão teórica, cujo objetivo é identificar como a GC pode contribuir no processo de co-criação de valor em serviços informacionais.

\section{VAlidaçÃo do ProKnow-C}

As palavras chaves são um ponto de relevância a ser analisado e que evidencia a efetividade do mapeamento de um tema por meio do ProKnow-C. No Portfólio Bibliográfico (PB) foram identificadas 89 palavras-chave, o qual pode-se observar por meio da Figura 1 aquelas que se repetiram por no mínimo duas vezes.

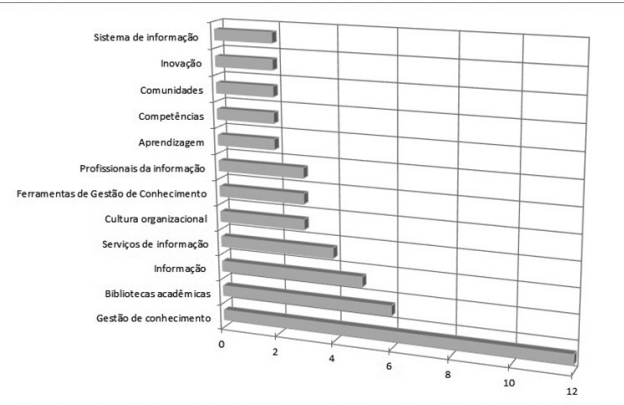

Figura 1. Palavras chaves do PB Fonte: elaborado pelos autores

A palavra-chave que mais se destacou foi Gestão do conhecimento, com 12 ocorrências, seguida de Bibliotecas acadêmicas com 6 ocorrências, Informação com 4, e Serviços de informação com 3. Pode-se considerar que houve aderência das palavras em relação ao tema da pesquisa, o que valida à utilização delas no processo de busca.

\section{Discussóes teóricas}

Esta seção aborda as discussões sobre algumas temáticas levantadas por meio da leitura do portfólio bibliográfico. Os assuntos elencados mais adiante referem-se a um aspecto levantado por Vargo e Lusch (2004) no que compete a GC nesta lógica. O ponto de partida da discussão é a afirmação de que 
a Lógica Dominante do Serviço, é vista como um modelo de troca centrado no serviço, enquanto troca de conhecimento, essa permuta é feita através de meios "intangíveis, competências, dinâmicas, processos de troca e relacionamentos e recursos operantes" (Vargo e Lusch, 2004: 15).

Assim, sob a ótica dos serviços informacionais, discutira-se sobre os recursos operantes (informação e conhecimento) e sua gestão; o processo de troca (aprendizagem organizacional); as competências (dos profissionais); e o valor caracterizado neste nesse novo contexto (co-criação).

\section{Recursos operantes: Gestão do Conhecimento e da Informação}

A captura, organização e disseminação do conhecimento são processos inerentes as duas áreas de pesquisas: Gestão do Conhecimento e Ciência da Informação, cujo contruto se diferenciam (informação e conhecimento), contudo, alinham-se no que tange seus beneficios para as organizações.

Serrat (2010) aborda em sua obra, os processos de capturar, organizar, disseminar o conhecimento. Essa rotina esta presente quando descreve sobre a arquitetura para aprendizagem, as ferramentas de gerenciamento do conhecimento, e o quadro de competência organizacional. Para o autor, as iniciativas de GC podem ser aplicadas para catalizar e facilitar os processos citados acima.

Em seu trabalho que abordou a questão entre Gestão da Informação (GI) e GC, Valentim (2008) corrobora com Serrat (2010) no sentido que a GC, entre outros fatores, refere-se a criação/geração, aquisição/apreensão, compartilhamento/socialização e uso/utilização de conhecimento.

Inobstante, é possível perceber que estes processos também estão envolvidos na GI. Valentim (2008) afirma que a GI em ambientes organizacionais visa, entre outros processos: prospectar, coletar, filtrar, monitorar, disseminar informações.

Claro que os objetos de pesquisa das áreas são distintos (informação e conhecimento), porém, é interessante observar que alguns processos são coincidentes, pois visão a utilização destes insumos, para o bem organizacional, contudo, com intuitos bem definidos.

O intuito da GC visa trabalhar a cultura organizacional/informacional e a comunicação organizacional/informacional em ambientes organizacionais, de forma a propiciar um ambiente positivo (Valentim, 2008). Já a GI objetiva apoiar o desenvolvimento das atividades/tarefas cotidianas e o processo decisório nesses ambientes (Valentim, 2004: 1).

Portanto, observa-se que a GC e GI estão preocupadas em gerenciar conhecimento e informação respectivamente, e que utilizam de mesmos processos para o desenvolvimento organizacional, porém, com distintas finalidades. 
Para melhor visualizar esta distinção, Valentim (2008), considera que a GI enfoca em fluxo formais do ambiente organizacional (o que esta sistematizado, formalizado, explicitado em qualquer suporte). A GC enfoca os fluxos informais do ambiente organizacional (o que não esta explicitado, formalizado, sistematizado) como, por exemplo: cultura, comunicação, comportamento, aprendizagem, valores, práticas. Segundo o autor a GC alimenta a GI, que retroalimenta a gestão do conhecimento, em um ciclo sem começo, meio e fim. Os indivíduos são os atores desses processos, cujo papel é fundamental para a dinâmica acontecer.

Serrat (2010) também descreve sobre o fluxo de conhecimento e afirma que isso faz parte da constituinte da auditoria do conhecimento. Mais uma vez observa-se que a dinâmica entre GI e GC se aproximam, em observar que dentro dos processos, ações como a análise de fluxo (informação e conhecimento), são utilizadas para melhor aproveitar este aporte para o desenvolvimento organizacional.

Acredita-se que a GI e a GC em ambientes organizacionais contribuem para o sucesso organizacional, do processo decisório, ao planejamento e às estratégias de ação.

O contexto atual, sua complexidade e pluralidade demandam atenção ao papel do conhecimento e informação nas organizações. A gestão destes insumos é extremamente necessária para o desenvolvimento de atividades, aprendizagem organizacional, praticas organizacionais, criatividade e inovação, de forma a reduzir o grau de incertezas em processos decisórios, e propiciar um ambiente organizacional dinâmico e sustentável economicamente.

\section{Aprendizagem organizacional: a Gestão da Informação}

O cenário em que estão dispostas as bibliotecas exigem esforços para adaptar a organização e aproveitar o conhecimento de seus profissionais para usufruto de sustentabilidade e dinamicidade. A aprendizagem é uma forma deste processo ser executado por meio da GC. Ela é observada quando o participante experimentam novas informações contextualizadas (aprendizagem), refletem sobre a as experiências (conscientização da aprendizagem) e aplicam conhecimentos vivenciados em novos contextos (transferência de aprendizagem). Neste processo, segundo Davis e Somerville (2006), os participantes melhoram a capacidade coletiva para identificar e formular perguntas, reunir e avaliar informações, organizar e sintetiza-las, e, finalmente, ter ideias para informar e aconselhar. Como consequência, as atividades de aprendizagem organizacional melhoraram o alinhamento institucional, melhoraram a estratégia e a integração funcional (Davis e Somerville, 2006). 
A GI subsidia o aprendizado organizacional. Serrat (2010) considera a informação, entre outros fatores, insumo para fortalecer a identidade, sustentar comunidades de aprendizagem, manter clientes e funcionários informados e conscientes do desenvolvimento corporativo, conectar pessoas e fornecer acesso aos seus conhecimentos e ideias etc... Cita da importância da comunicação, no que tangem a transferência lateral de informação e conhecimento.

Assim, observa-se ponderadamente, o uso da informação nos processos de aprendizagem organizacional. Contribuindo para esta abordagem, Ferreira e Duarte (2012) ratificou a importância da informação como elemento substancial para as ações da aprendizagem organizacional, posto que seja ela que transfere e desperta relevância, muda o curso das ações, unifica interesses e proporciona uma busca contínua pelo aprendizado. Os autores ainda afirmam que é mediante a informação que a aprendizagem organizacional é construída pelos ativos intangíveis e se configura como fator essencial ao desenvolvimento de uma organização.

Para Ferreira e Duarte (2012), a informação é elemento primeiro para formação do conhecimento, isto é, o capital intelectual da organização é construído mediante o uso da informação, que visa angariar sucesso no processo de ensino-aprendizagem, nas organizações voltadas para a aprendizagem, na definição e na busca de objetivos. Assim, melhor que dispor a informação como recurso para o sucesso organizacional, sua gestão propicia um melhor aproveitamento deste recurso, direcionamento a organização para o sucesso.

A GI despende como recurso estratégico para diversos objetivos organizacionais, apoia a tomada de decisão, sustenta planejamentos, direciona esforços etc... Na aprendizagem organizacional, junto com o conhecimento, é observada quando os indivíduos passam a incorporar os elementos informacionais, inovando e tecendo uma cultura capaz de valorizar o conhecimento na busca pelo crescimento organizacional (Ferreira e Duarte, 2012).

Assim, bibliotecas que fazem uso da GC, precisam aproveitar sua experiência em gerenciar a informação para subsidiar e melhorar os processos de aprendizagem organizacional. Estudos mostraram que as bibliotecas, possuem atributos diferenciados (GI) para se beneficiarem e poderem dispor de novos e melhores serviços, baseados nos conhecimentos de seu corpo profissional.

\section{Profissionais da informação: competências em conhecimento e em informaçâo}

A aprendizagem organizacional permite por meio de seus profissionais, aprender, conscientizar, e transferir o conhecimento para a instituição, contudo, quanto maior suas competencias em conhecimento e informação, melhores são os resultados da GC. 
Para Serrat (2010) competência é o estado ou qualidade de adequadamente ou qualificadamente entregar uma tarefa específica, ação ou função com sucesso. Portanto, vincula-se o conceito, a ideia de melhor atender as necessidades organizacionais. O autor afirma que a competência para conhecimento é uma gama especifica de conhecimento e habilidades utilizados para melhorar o desempenho organizacional.

Ainda segundo Serrat (2010), a competência em conhecimento se faz antes, durante e após um evento. É preciso competências para as haver "soluções de conhecimento". Essas competencias estão relacionadas em áreas como: desenvolvimento de estratégia, técnicas de gestão, mecanismos de colaboração, compartilhamento de conhecimento e aprendizagem, e captura e armazenamento de conhecimento.

A competência em informação é definida como um processo contínuo de internalização de fundamentos conceituais, atitudinais e de habilidades necessário à compreensão e interação permanente com o universo informacional (Fidelis e Barbosa, 2012). Já Zuccari e Belluzzo (2016) ressaltam que para as pessoas manterem-se em constante aprendizagem elas precisam ser competentes em informação, o que significa saber reconhecer quando uma informação é necessária para a sua vida, para isso ela deve ser capaz de localizar, avaliar e usar efetivamente a informação, ou seja, saber como devem aprender.

Em seu olhar para competência informacional no contexto organizacional, Fidelis e Barbosa (2012) afirmam que apesar de ser um fenômeno abstrato e intangível, a informação tornou-se um recurso primordial ao desenvolvimento de qualquer organização. Ter competência para lidar com este recurso e assim proporcionar um melhor desempenho organizacional é a habilidade que todo profissional tem que buscar nos dias de hoje. Entender os contextos de atuação e usar a informação para determinar o caminho a ser seguido é a habilidade necessária que definirá o sucesso ou não da organização.

Diante o exposto, observa-se uma sinergia entre as "competencias" para o desenvolvimento organizacional, principalmente no que remete a aprendizagem.

Sob o ponto de vista da GC, Serrat (2010) delimita as areas de competência e suas respectivas soluções de conhecimento. Uma dás areas abordadas refere-se a "partilha de conhecimento", "aprendizado" e "estratégia para aprendizado organizacional". Neste contexto, o autor traz questionamentos sobre o uso da informação na GC, ao abordar sobre a problemática de sobrecarga de informação e a necessidade de demostrar o conhecimento, bem como, a dinâmica de armazenamento de informações sobre conhecimento, habilidades, experiencias e interesses de pessoas. Nesse aspecto, observa-se o uso GI para com a GC, e pode-se inferir que ter competências informacionais pode ser uma das respostas para estes questionamentos. 
Sob o ponto de vista da GI, Melo e Araújo (2007) afirmam que o conceito de competência informacional esta relacionado com as atitudes que facilitam criar e compartilhar conhecimento, ou seja, aprendizado. Considera competência informacional um subconjunto do aprendizado.

Em relação a competência informacional e a competência em conhecimento, acredita-se que uma alicerça a outra, pois se inter-relacionam constantemente. As responsabilidades da GC esta embasada em habilidades para lidar com informação, ou seja, competência informacional (Melo e Araújo, 2007).

Os profissinais da inormação devem ser capacitados para atuarem de forma eficiente e efizar para melhor usufruir de suas competencias e pensar a biblioteca dinamica, que atendam as necessidades de seus usuários. A nova dinamica organizacional, como visto aqui, exige organizações adaptadas as mudanças, aos interesses cada vez mais volateis de seus usuários, aos anseios e ansiedades por serviços informacionais que atendam suas necessidades em um universo informacional complexo. É preciso olhar para fora da organização, e perceber o quanto o usuário pode colaborar para que as bibliotecas agregem valor aos seus serviços, e os usuários se beneficiem com isso.

\section{Co-criação de valor e a Lógica Dominante em Serviços}

Walters e Lancaster (1999) referem-se à valor como atributos à produtos e serviços. Uma questão levantada pelos autores foi a dinamica organizacional sobre a visão da organização estar voltada para os clientes. Em sua conclusão, os autores trazem uma nova dinâmica a ser abordada. No novo milênio, os pontos de vista de valor e satisfação devem se fundir, e considerar o termo coprodução como um novo vocabulário do marketing (Walters e Lancaster, 1999: 655).

Koetz e Koetz (2012) abordam em seu estudos, os avanços, no que tange a co-criação de valor em termos de produtos e serviços. Vale resaltar que a obra das autoras aborda a temática da Lógica Dominante em Serviços (SDL), inobstante, um dos pontos desta logica refere-se a co-criação de valor entre a organização e o cliente. E é sobre esta ótica que parte a discussão.

Resumidamente, a SDL proposta por Vargo e Lusch (2004) dita que toda economia é uma economia de serviços, e assim, é orientada para recursos operante (conhecimento e habilidade) e para a co-criação de valor entre empresa e cliente. Segundo os autores, o conceito de valor era centrado em produtos, o qual era identificado como valor acrescentado ao produto no processo de produção, elemento principal no momento da troca, representado pelo valor em troca. No novo modelo centrado em serviços, o valor somente pode ser criado e determinado pelo usuário no processo de consumo, o valor 
em uso. Assim, a co-criação de valor refere-se a co-produção, isto é, a participação do cliente ou parceiros na cadeia de valor na criação da propria oferta (Koetz e Koetz, 2012).

Desta forma, observa-se que o processo de co-criação de valor, é fruto de uma realidade onde o cliente participa do processo de criação de valor, beneficiando a empresa que fornece produtos e serviços, e o cliente que compra e usa produtos e serviços.

Outro ponto, refere-se à “informação", isto é, a informação do ponto de vista de Walters e Lancaster (1999) no tocante a cadeia de valor. Segundo os autores, a gestão da informação tem papel importante na cadeia de valor. A informação é um facilitador no desenvolvimento de novos produtos e na satisfação de cliente. Ela é utilizada em sistemas de informação, e é direcionadora de vantagens competitivas.

Nesta perspectiva, Koetz e Koetz (2012) também abordam a tematica informação em seu estudo. As autoras, sob a otica da SDL afirmam que a informação, alem de habilidades, conhecimento, interatividade e conectividade, estão contidos neste modelo cuja enfase esta nos aspectos intagiveis. Segundo o estudo, a informação tem papel relevante na nova economia, onde as tecnologias de informação e comunicação transformaram a sociedade. $\mathrm{O}$ fluxo de informação que era fisico, se transformou em digital, cuja velocidade, relações, acessibilidade, mudaram o contexto das organizações e pessoas, no que tange seu uso.

Observa-se que informação tem papel relevante no processo de criação de valor pelas organizações, ela é o insumo para tomadas de decisões, e esta relacionada com o direcionamento da SDL e consequentemente na proposta de co-criação de valor.

Em bibliotecas não deve ser diferente. Islam, Agarwal e Ikeda (2015) em seu estudo sobre a criação de um quadro conceitual preliminar sobre o fenómeno da co-criação de valor de serviços em bibliotecas, propôs integrar sistemicamente os diferentes intervenientes, neste caso a biblioteca e os seus clientes, com as suas estruturas cognitivas, comportamentais e emocionais numa rede complexa de interligações.

Para os autores, esta co-criação de valor conjunta entre a biblioteca e o usuário cria as condições para a inovação de serviços, onde a biblioteca e o usuário estão trabalhando juntos para mudar, melhorar e criar algo novo e valioso. Este quadro proposto incorpora vários fluxos de trabalho dentro da SDL é aplicado em biblioteca. Ele combina as funções e responsabilidades das três esferas - a biblioteca, o usuário, e sua interação para co-criar valor.

Agarwal e Marouf (2014) consideram que a gestão do conhecimento poderia também servir como um modelo possível para implementar co-criação de valor nas bibliotecas universitárias. 
Islam, Agarwal e Ikeda (2015) afirmam que as bibliotecas acadêmicas precisam mudar sua ênfase da criação de valor para a co-criação e convidar professores / alunos / pessoal / investigadores para tomar parte ativa no processo de desenvolvimento de serviços. Eles precisam permitir que as comunidades de usuários a pensar em si mesmos como co-criadores dos serviços que utilizam.

A mudança de um paradigma baseado em bens tangíveis para uma lógica baseada em ativos intangíveis, em um cenário global inovador e dinâmico, pode levar as organizações a enfrentar uma série de situações inesperadas (Bhatt, 2001). Nessas situações, até mesmo o conhecimento tácito, considerado como uma das bases para a competitividade, pode se tornar obsoleto (Ambrosini e Bowman, 2001).

Nesse cenário, o arcabouço teórico e prático oferecido pela GC é de grande valia. Como descreve Bhatt (2001: 73), uma das formas de trabalhar essas situações inesperadas é justamente coordenar os padrões de interação nas organizações.

\section{Conclusĩo}

Este estudo teve como objetivo identificar como a Gestão do Conhecimento (GC) pode contribuir no processo de co-criação de valor em serviços informacionais. Com o uso da ferramenta de intervenção ProKnow-C foi possível selecionar um portfólio bibliográfico representativo que por meio da metodologia proposta, possibilitou a discussão de quatro aspectos da GC no tocante aos serviços informacionais.

As discussões sobre esses aspectos consideraram a obra de Vargo e Lusch (2004) no que compete a GC e seus benefícios organizacionais no tocante as competências, dinâmicas, processos de troca e relacionamentos e recursos operantes.

Assim, sob a ótica dos serviços informacionais, discutiu-se sobre os recursos operantes (informação e conhecimento) e sua gestão; o processo de troca (aprendizagem organizacional); as competências (dos profissionais); e o valor caracterizado neste nesse novo contexto (co-criação).

O primeiro aspecto retratou sobre a informação e conhecimento, os recursos operantes conforme Vargo e Lush (2004). Percebeu-se que a análise destes fundamentos de GC e Gestão da Informação (GI), pautadas pela visão de processos igualitários (captura, organização e disseminação) estão relacionados a fenômenos que são representativos para os avanços na área da Ciencia da Informação, bem como, para a GC. Reconhecer estas 
especificidades, as dinamicas que regem estes processos, podem permitir novos estudos ao aproximar o carater interdisciplinar das áreas em questão, e beneficiar as bibliotecas no que tange os serviços informacionais, e a dinamica para abordar a Lógica Dominante em Serviços (SDL), uma vez que estes recursos, estão contidos nestas instituições.

Sobre o segundo aspecto, a GI como subsidio para aprendizagem organizacional. A GI visa contribuir na GC e consequente o aprendizado organizacional. Isso porque, muitas vezes é desprezado esse capital (informação) por falta de modelos norteadores das ações que buscam a sobrevivência e inovação organizacional, prejudicando, a formação do capital intelectual, e consequentemente, sua gestão para o desenvolvimento organizacional.

O terceiro aspecto, competencia, conclui-se que o diálogo demonstrou a relevância entre competência em informação e competência em conhecimento, de forma a observar que ambas são prioritárias para o desenvolvimento organizacional, e estão inter-relacionadas, com propósitos distintos, mas com fins de almejar vantagens competitivas.

No tocante ao ultimo aspecto, a co-criação de valor e a SDL, conclui-se que temática proposta por Walters e Lancaster (1999), seguiu por caminhos proficuos, e que a SDL, descrita por Vargo e Lusch (2004) é um exemplo, ao considerar a co-criação de valor como elemento principal desta nova logica. A obra de Koetz e Koetz (2012), trouxe o fundamento dos aspectos aqui propostos, (valor e informação), permitindo uma explanação mais atual da temática, por se tratar exatamente da mudança paradigmatica ou não da SDL, e o quanto o valor é caracterizado neste novo contexto.

Por fim, acredita-se que o modelo dos serviços de uma biblioteca nunca serão mais o mesmo, pois as dinamicas tecnologicas mudaram vários paradigmas. O desafio maior é que novas filosofias de gestões, com a GC, possam oferecer ferramentas para melhor prestar serviços informacionais, e não somente agregar valor, mais sim, co-criar valor, para que ambos se beneficiem neste contexto.

Cabe ressaltar as limitações desta pesquisa: i) restrita a artigos publicados em revistas científicas indexadas nas bases de dados da CAPES; ii) julgamento e interpretação dos autores em relação à análise dos Portfólio Bibliográfico $(\mathrm{PB})$, portanto a interpretação pode não corresponder aos ideais dos autores originais dos periódicos. Sugere-se, como trabalho futuro, a possibilidade de pesquisas em novas e diferentes bases de dados, distintas características e novos trabalhos que proporcionem a redução de possíveis gaps deste estudo. 


\section{REFERÊNCIAS}

Agarwal, N. K. e L. N. Marouf. 2014. "Initiating knowledge management in colleges and universities: A template". International Journal of Knowledge Content Development E Technology 4 (2): 67-95. http://dx.doi.org/10.5865/IJKCT.2014.4.2.067

Ahmad, K. 2017. "The Perspective of Library and Information Science (LIS) Professionals Toward Knowledge Management in University Libraries". Journal of Information \& Knowledge Management (JIKM) 16 (02): 1-11. https://doi.org/10.1142/S0219649217500150

Alavi, M., T. R. Kayworth e D. E. Leidner, 2005. "An empirical examination of the influence of organizational culture on knowledge management practices". Journal of management information systems 22 (3): 191-224. https://doi.org/10.2753/MIS0742-1222220307

Ambrosini, V. e C. Bowman. 2001. "Tacit knowledge: Some suggestions for operationalization”. Journal of Management studies 38 (6): 811-829. https://doi.org/10.1111/1467-6486.00260

Balagué, N., P. Düren e J. Saarti. 2016. “Comparing the knowledge management practices in selected European higher education libraries". Library Management 37 (4-5): 182-194. https://doi.org/10.1108/LM-12-2015-0068

Balaji, B. P., M. S. Vinay, B. G. Shalini e J. S. Mohan Raju 2018. "An integrative review of Web 3.0 in academic libraries". Library Hi Tech News 35 (4): 13-17. https://doi.org/10.1108/LHTN-12-2017-0092

Bhatt, G. D. 2001. "Knowledge management in organizations: examining the interaction between technologies, techniques, and people". Journal of knowledge management 5 (1): 68-75. https://doi.org/10.1108/13673270110384419

Botelho, M. A., A. M. Monteiro e V. Valls. 2007. “The sporting knowledge management: An experience of Seme library”. Ciência da Informação 36 (1): 175-188. https://doi.org/10.1590/S0100-19652007000100014

Carvalho, M. M. M., J. R. Menezes e C. Domingues, 2012. "A co-criação de valor nos serviços bibliotecários: uma abordagem conceptual em construção". Trabalho apresentado em XXII Jornadas Luso-Espanholas de Gestão Científica, Bragança, Portugal, 13 fevereiro,.

Costa, B. K. e P. L. Martins. 2012. “Co-criação de Valor através da Gestão do Conhecimento: um Estudo da Empresa Subway do Setor Fast Food”. Trabalho apresentado em Simpósio de Excelência em Gestão e Tecnologia, Resende, Rio de Janeiro, Brasil, 24, 25 e 26 de outubro.

Creswell, J. W. 2014. Research Design: Qualitative, Quantitative, and Mixed Methods Approaches. CA: Sage Publications.

Davis, H. L. e M. M. Somerville. 2006. "Learning our way to change: Improved institutional alignment”. New Library World 107 (3-4): 127-140. https://doi.org/10.1108/03074800610654907

Dickinson, N. 2004. "Responding to the information challenges”. 21 (2): 92-97. https://doi.org/10.1177/0266382104044723 
Ensslin, L., S. R. Ensslin e H. M. Pinto. 2013. "Processo de investigação e Análise bibliométrica: Avaliação da Qualidade dos Serviços Bancários”. Revista de Administração Contemporânea 17 (3): 325-349.

Ferguson, S., P. Hider e A. Lloyd. 2008. "Are librarians the ultimate knowledge managers? A study of knowledge, skills, practice and mindset”. The Australian Library Journal 57 (1): 39-62.

https://doi.org/10.1080/00049670.2008.10722440

Ferreira, T. E. D. L. R. e E. N. Duarte, 2012. “A adoção da informação na gestão da aprendizagem organizacional no campo da Ciência da Informação”. Perspectivas em Gestão E Conbecimento 2 (2): 87-103.

Fidelis, J. R. F. e R. R. Barbosa. 2012. "A competência informacional e sua influência na percepção de variáveis organizacionais estratégicas em IES privadas”. Perspectivas em Gestão \& Conbecimento 2 (2): 27-39.

Fowell, S. 2002. "Bridging the gap between information resource design and enterprise content management". In International conference on Asian digital libraries, 507-515. Berlin, Heidelberg: Springer.

Islam, M. S., M. A. K. Siddike, S. Nowrin e S. Naznin. 2015. "Usage and applications of knowledge management for improving library and information services in Bangladesh”. Journal of Information e Knowledge Management 14 (03). https://doi.org/10.1142/S0219649215500264

Islam, M. A., N. K. Agarwal e M. Ikeda. 2015. "Conceptualizing value co-creation for service innovation in academic libraries”. Business Information Review 32 (1): 45-52. https://doi.org/10.1177/0266382115573155

Johannsen, C. G. 2000. "Total quality management in a knowledge management perspective”. Journal of Documentation 56 (1): $42-54$.

Koetz, C. I. e C. M. Koetz. 2012. "A lógica dominante do serviço em marketing: um novo paradigma?”. Revista Estudos do CEPE (Santa Cruz do Sul) 36: 147-169. https://doi.org/10.17058/cepe.v0i0.3118

Melo, A. V. C. D. e E. A. D. Araújo. 2007. "Competência informacional e gestão do conhecimento: uma relação necessária no contexto da sociedade da informação". Perspectivas em Ciência da Informação 12 (2): 185-201. https://doi.org/10.1590/S1413-99362007000200012

Miao, Q. 2001. "From literature center to knowledge portal: Shanghai Library in search of excellence 2.0". Library Review 50 (7-8):349-354. https://doi.org/10.1108/EUM0000000006072

Pantry, S. e P. Griffiths. 2000. "Being an intrapreneur and creating a successful information service within your organization”. Business information review 17 (4): 205-214. https://doi.org/10.1177/0266382004237773

Pérez Rodríguez, Y. e Y. Milanés Guisado. 2008. "La biblioteca universitaria: reflexiones desde una perspectiva actual". Acimed 18 (3).

Roy, B. 1993. "Decision science or decision-aid science?”. European journal of operational research 66 (2): 184-203. https://doi.org/10.1016/0377-2217(93)90312-B

Serrat, Olivier. 2010. "Notions of Knowledge Management". In Asian Development Bank. Knowledge solutions: tools, methods, and approaches to drive development forward and enhance its effects. Mandaluyong City, Philippines: Asian Development Bank. 
Shanhong, T. 2002. "Knowledge management in libraries in the 21st century", in Libraries in the information society, T. V. Ershova e Y. E. Hohlov (eds.). IFLA publications, 102. München: K.G. Saur.

Ugwu, C. I. e J. N. Ekere. 2018. "The role of knowledge management in providing innovative services in university libraries in Nigeria". Global Knowledge, Memory and Communication 67 (6/7): 350-376. https://doi.org/10.1108/GKMC-10-2017-0086

Ugwu, C. I. e J. N. Ekere. 2019. "Knowledge management for improving services in federal university libraries in Nigeria". Journal of Librarianship and Information Science 51 (2): 356-369. https://doi.org/10.1177/0961000617742446

Valentim, M. L. P. 2004. Gestão da informação e gestão do conhecimento: especificidades e convergências. Londrina: Infohome.

Valentim, M. L. P. 2008. "Gestão da Informação e Gestão do Conhecimento em ambientes organizacionais". Tendências da Pesquisa Braileira em Ciência da Informação 1 (1): 1-16.

Van Deventer, M. J. e R. Snyman. 2004. "Measuring for sustainability: A multi-dimensional measurement framework for library and information services". Libri 54 (1): $1-8$.

Vargo, S. L. e R. F. Lusch. 2004. "Evolving to a new dominant logic for marketing”. Journal of marketing 68 (1): 1-17.

Venkatesh, N. e S. Kalpavalli. 2014. "Building Knowledge Management-based Systems: Initiatives at Research Centre Imarat”. DESIDOC Journal of Library e Information Technology 34 (4): 304-308. https://doi.org/10.14429/djlit.34.5841

Walters, D. e G. Lancaster. 1999. "Value and information: concepts and issues for management". Management Decision 8 (37): 643-656. https://doi.org/10.1108/00251749910291613

Wijnhoven, F. e J. Kraaijenbrink. 2008. "Product oriented design theory for digital information services”. Internet research 18 (1): 93- 120. https://doi.org/10.1108/10662240810849612

Zuccari, P. e R. C. B. Belluzzo. 2016. "A competência em informação e o perfil empreendedor no âmbito das organizações". Perspectivas em Gestão \& Conhecimento $6: 61-71$.

\section{ApÊndice i. Portfólio Bibliográfico}

Alavi, M., T. R. Kayworth e D. E. Leidner. 2005. "An empirical examination of the influence of organizational culture on knowledge management practices". Journal of management information systems 22 (3): 191-224.

Balagué, N., P. Düren e J. Saarti, 2016. “Comparing the knowledge management practices in selected European higher education libraries". Library Management 37 (4-5): 182-194.

Balaji, B. P., M. S. Vinay, B. G. Shalini e J. S. Mohan Raju. 2018. “An integrative review of Web 3.0 in academic libraries". Library Hi Tech News 35 (4): 13-17.

Botelho, M. A., A. M. Monteiro, e V. Valls. 2007. "The sporting knowledge management: An experience of Seme library”. Ciência da Informação 36 (1): 175-188. 
Chen, J., J. Guo, R. Shuhuai, S. Xingjun, L. Haiqing e C. Jialin. 2009. “From information commons to knowledge commons: Building a collaborative knowledge sharing environment for innovative communities". The Electronic Library 27 (2): 247-257.

Chiu, T. H. 2005. "Attributes and factors affecting the organization of knowledge resources: A case study of the library and information service industry in Taiwan". Ko Knowledge Organization 32 (3): 128-134.

Chou, T. C., P. L. Chang, Y. P. Cheng e C. T. Tsai. 2007. "A path model linking organizational knowledge attributes, information processing capabilities, and perceived usability". Information \& Management 44 (4): 408-417.

Chou, T. C., P. L.Chang, C. T. Tsai e Y. P. Cheng. 2005. "Internal learning climate, knowledge management process and perceived knowledge management satisfaction". Journal of information science 31 (4): 283-296.

Davis, H. L. e M. M. Somerville. 2006. "Learning our way to change: Improved institutional alignment”. New Library World 107 (3-4): 127-140.

Dickinson, N. 2004. "Responding to the information challenges". Business Information Review 21 (2): 92-97.

Ferguson, S., P. Hider e A. Lloyd. 2008. "Are librarians the ultimate knowledge managers? A study of knowledge, skills, practice and mindset”. The Australian Library Journal 57 (1): 39-62.

Fowell, S. 2002. "Bridging the gap between information resource design and enterprise content management". In International conference on Asian digital libraries, 507-515. Berlin, Heidelberg: Springer.

Gray Southon, F. C., R. J. Todd e M. Seneque. 2002. "Knowledge management in three organizations: an exploratory study". Journal of the American society for Information Science and Technology 53 (12): 1047-1059.

Husain, S., e M. Nazim. 2015. "Use of different information and communication technologies in Indian academic libraries". Library Review 64.

Islam, M. S., M. A. K. Siddike, S. Nowrin e S. Naznin. 2015. "Usage and applications of knowledge management for improving library and information services in Bangladesh”. Journal of Information e Knowledge Management 14 (03).

Johannsen, C. G. 2000. "Total quality management in a knowledge management perspective". Journal of Documentation 56 (1): $42-54$.

Joint, N. 2001. "Designing interfaces for distributed electronic collections: the lessons of traditional librarianship". Libri 51 (3): 148-156.

McManus, D. e B. Loughridge. 2002. "Corporate information, institutional culture and knowledge management: a UK university library perspective". New Library World 103 (9): 320-327.

Melnikova, E. V. 2014. "Enhanced functions of the modern STI system in Russia in the context of its improvement and of the development of the innovative orientation". Scientific and Technical Information Processing 41(1): 29-32.

Miao, Q. 2001. "From literature center to knowledge portal: Shanghai Library in search of excellence 2.0". Library Review 50 (7-8):349-354.

Pantry, S. e P. Griffiths. 2000. "Being an intrapreneur and creating a successful information service within your organization”. Business information review 17 (4): 205-214. 
Tanloet, P. e K. Tuamsuk. 2011. “Core competencies for information professionals of Thai academic libraries in the next decade (AD 2010-2019)". The International Information E Library Review 43 (3): 122-129.

Ugwu, C. I. e J. N. Ekere. 2018. “The role of knowledge management in providing innovative services in university libraries in Nigeria". Global Knowledge, Memory and Communication 67 (6/7): 350-376.

Ugwu, C. I. e J. N. Ekere. 2019. "Knowledge management for improving services in federal university libraries in Nigeria". Journal of Librarianship and Information Science 51 (2): 356-369.

Van Deventer, M. J. e R. Snyman. 2004. "Measuring for sustainability: A multi-dimensional measurement framework for library and information services". Libri 54 (1): $1-8$.

Venkatesh, N. e S. Kalpavalli. 2014. "Building Knowledge Management-based Systems: Initiatives at Research Centre Imarat”. DESIDOC Journal of Library e Information Technology 34 (4): 304-308.

Wijnhoven, F. e J. Kraaijenbrink. 2008. "Product oriented design theory for digital information services”. Internet research 18 (1): 93-120.

Para citar este texto:

Silveira, Murilo Mauro y Gregório Varvakis. 2021. "Gestão do Conhecimento e co-criação de valor em Serviços Informacionais”. Investigación Bibliotecológica: archivonomía, bibliotecología e información 35 (86): 73-97.

http://dx.doi.org/10.22201/iibi.24488321xe.2021.86.58255 